\title{
An approach to the interpretation on intensional contexts
}

\author{
Elena D. SMIRNOVA ${ }^{1}$
}

\begin{abstract}
The paper introduces a non-standard analysis of intensional contexts on the ground of generalized approach to semantics construction. The principles of building such kind semantics are consider. As far as I can see it is an idea on domains and anti-domains that lays in the ground a semantics of intensional contexts. Intensional contexts differ from extensional by ascription of specific values to intensional predicates (operators) and, what is more important, by a way of their combination with arguments. Thus constructing operations play the leading role in proposed analysis. The peculiarities of IPL: any expression including intensional predicates and operators has an intension as well as an extension.
\end{abstract}

Keywords: generalized semantics, domains and anti-domains, propositional concept, operation of abstraction

The paper introduces a non-standard analysis of intensional contexts on the ground of generalized approach to semantics construction. In so doing, an expressive power of the natural language appropriate for the representation of intensional context's logical structure is considered.

A logical structure of an intensional context is determined by both interpretation of intensional signs and accepting a specific applicative operation of intensional operators (predicates) to terms. This procedure defines an algorithm, which allows finding extensions and intensions of corresponding contexts.

It is a set of laws, presuppositions and conventions $\Gamma$ that being accepted determines states of affairs in possible worlds semantics.

\footnotetext{
${ }^{1}$ This work is supported by Russian Foundation for Humanities, grant № 11-03-00143.
} 
When epistemic contexts such as $\mathbf{B} a(p)$ considered, these principles and conventions depend on a subject $a$, denoted as $\Gamma a$. They may be partially or completely agree with the laws of a theory, that is $\Gamma a \subseteq$ $\Gamma$ or $\Gamma a \cap \Gamma$ and so on (where $\Gamma a=\Gamma$ is the case of omniscience). If $\Gamma a \neq \Gamma$, a subject can 'break the laws' of a theory, because they are not included in $\Gamma a$. To this extent the subjective worlds $\mathrm{WP}^{\circ}$ may be imaginary, and give rise to contradictions and paradoxes. This idea is to be taken into account when argumentation and the process of conviction are considered.

A semantical analysis of epistemic contexts generate a bulk of questions. What is possible interpretation of epistemic operators? What are the truth-conditions for epistemic statements with such operators? What is their logical structure? In what follows we will focus on a method of interpretation of intensional signs (operators and predicates) on the ground of a generalized approach to semantics construction.

We consider that adequate semantics may be constructed without using the concepts of contradictory or incomplete state descriptions. In any case, these concepts are not taken as a background and no assumptions are made in relation to the objects of discourse.

Instead partially defined predicates are accepted. We consider that predicates of truth, falsity belong to this kind - they can be partially defined. Second, we proceed from the idea of the symmetry of concepts of truth and falsity (and this is very important). Falsity is considered to be an independent notion and not as absence or negation of the truth.

Let us consider the principles of building language semantics. I shall construct my semantics using the notion of possible worlds. Let $W$ be a non-empty set of possible worlds, $\varphi$ a function ascribing a pair of sets $\left\langle H_{1}, H_{2}\right\rangle$ to propositional variables where $H_{1} \subseteq W$, $H_{2} \subseteq W$.

$\varphi_{T}(p)=H_{1}$ is the class of worlds in which $p$ holds (the domain of $p$ ).

$\varphi_{F}(p)=H_{2}$ is the class of worlds in which $p$ does not hold (the anti-domain of $p$ ).

The function of ascribing values to propositional variables is given in a generalized form: not the truth values in a given world, that is, 
not the objects $t$ and $f$, are ascribed to propositional variables, but special 'intensional objects' - classes of worlds $\varphi_{T}(p)$ and $\varphi_{F}(p)$. It is this that gives the intensional character to the propositional connectives, cf. [3, V].

We shall use a propositional language with the logical connectives $\&, \vee, \supset, \sim$. Let us introduce conditions of ascribing truth values to complex formulas as follows:

$$
\begin{aligned}
\varphi_{T}(A \& B)=\varphi_{T}(A) \cap \varphi_{T}(B), & \varphi_{F}(A \& B)=\varphi_{F}(A) \cup \varphi_{F}(B), \\
\varphi_{T}(A \vee B)=\varphi_{T}(A) \cup \varphi_{T}(B), & \varphi_{F}(A \vee B)=\varphi_{F}(A) \cap \varphi_{F}(B), \\
\varphi_{T}(\sim A)=\varphi_{F}(A), & \varphi_{F}(\sim A)=\varphi_{T}(A)
\end{aligned}
$$

When defining logical connectives, no limitations are imposed on the relations between the classes $\varphi_{T}(A)$ and $\varphi_{F}(A)$. The independence in ascribing domains and anti-domains to propositions allows us to treat the operation of negation in a generalized way. As a result of the above mentioned principles we get semantics with truth value gaps and with glut evaluations.

Dealing with such objects as the classes $\varphi_{T}(A)$ and $\varphi_{F}(A)$ it is possible to establish different relations between them, to accept or not to accept conditions (1) and (2). It is possible to accept one of them and reject the other, for they are independent of one another.

The relation between the classes $\varphi_{T}(A)$ and $\varphi_{F}(A)$ may but need not satisfy the following conditions:

(1) $\varphi_{T}(A) \cap \varphi_{F}(A)=\varnothing,(2) \varphi_{T}(A) \cup \varphi_{F}(A)=W$.

Accepting both (1) and (2) we get the standard semantics. Accepting (1) and rejecting (2) - shortly (1), (2) - semantics with truth value gaps; accepting (2) and rejecting (1) - semantics with glut evaluations (which permits of the overlap of truth and falsity); rejecting both (1) and (2) we get relevant semantics.

If both conditions (1) and (2) are accepted, the class of tautological formulas coincides with the class of irrefutable formulas and is identical to the class of tautologies of classical logic.

One of the peculiarities of analysis of intensional contexts is connected with an interpretation of intensional signs. This interpretation presupposes introduction of very special objects and leads to multiplication of abstract entities within semantical analysis. In 
other words this interpretation entails certain 'intensional' ontology. Let us examine what are the entities introduced this way. As far as I can see it is an idea of domains and anti-domains that lays in the ground of the semantics of intensional contexts.

A reference of an expression in a certain world represents its extension. If, following Carnap, we interpret intension as those entities that two $L$-equivalent statements $A$ and $B$ have in common, then they appear to be their domains: $\varphi_{T}(A) \equiv \varphi_{F}(A)$. An intension of a statement is often called its proposition or propositional concept.

Let $\mathbf{s}$ means a domain of a proposition $-\varphi T(A)$, and $\overline{\mathbf{s}}-$ its anti-domain, where $s \in 2^{W}$ and $s \subseteq W$.

Now we turn to a set $h$, whose elements are propositions $h=\left\{\mathbf{s}_{1}, \ldots, \mathbf{s}_{l}\right\}$. Hence, the domains are represented by families of propositions. Consider possible interpretations of modal operators and intensional predicates. Let their references be functions or relations defined on domains of statements or on families of such domains. Then the assignments are as follows.

Let $M$ be intensional operator (for example $\square p$ ) of the type $s / / s$, then possible referents are:

I $\left(2^{W}\right)^{\left(2^{W}\right)}$, where $2^{W}$ is a propositional concept (intension) $-\mathbf{s}$, i.e. $\varphi_{T}(p)$;

II $2^{W \times 2^{W}}-$ a relation $G ;\left\langle w_{i}, \mathbf{s}_{i}\right\rangle \in G$.

If we want an intensional sign to have both intension and extension, then we chose

III $\left(2^{\left(2^{W}\right)}\right)^{W}$, that is $f: W \rightarrow 2^{\left(2^{W}\right)}$, i.e. $w_{i} \rightarrow\left\{\mathbf{s}_{1}, \ldots, \mathbf{s}_{n}\right\}$ is an intension $\mathbf{M}$, while its extension in a world $w_{i}$ is $f\left(w_{i}\right)=h$, that is a a set of propositional concepts, where $w_{i} \in s_{i} ; s_{i} \in h ; h \in 2^{\left(2^{W}\right)}$.

An interpretation I represents the approach of $D$. Scott, and an interpretation II corresponds to R. Montague. The main advantage of the IIId approach lies in possibility to assign not only intensions or extensions but both of them as well.

An interpretation of intensional predicates is analogous [3, p. 246].

Now consider a structure of intensional contexts. This structure is determined by an interpretation of intensional signs, and first of all by the type of intensional entities assigned.

Logical aspect of analysis of intensional contexts is important for us. A key to the puzzle of these contexts can be found just there not 
in behavior of proper names. The core idea of this approach is that semantical analysis of intensional contexts presupposes, first of all, identification of peculiarities of their logical structure. What is the way in which components are linked in contexts that contain intensional predicates and operators? Thus constructing operations play the leading role in proposed analysis. Let us construct a semantics for Intensional Predicate Logic (IPL).

The language of IPL is based on the theory of semantical categories but the notion of index of category is extended: (1) $n$ and $s$ are indexes of categories ( $n$ is a category of singular terms; $s$ is a category of sentences); (2) if $\alpha$ and $\beta$ are indexes of categories then $\alpha / \beta$ and $\alpha / / \beta$ are indexes of categories. All categories of the type $\alpha / \beta$ are extensional and those of type $\alpha / / \beta$ are intensional. The method of interpretation for these two types of categories is especially important.

Let a model structure be the construction $\langle W, N, U, I, \Psi\rangle$ where $W$ is a non-empty set of worlds, $N$ is a set of normal worlds $(N \subseteq$ $W), U$ is a non-empty scope of individuals, $\Psi(H)$ is a non-empty scope of the possible world and $I$ is a function of interpretation.

1. If $P$ is a predicate expression of the category $s / n$, then $I(P)$ is an object of the type $\left(2^{U}\right)^{W}$.

2. If $\mathbf{Q}$ is a predicate of the category $s / / n$ then $I(\mathbf{Q})$ is an object of the type $\left(2^{\left(U^{W}\right)}\right)^{W}$ (similarly for $n$-placed predicates).

3. An object of the type $\left(2^{\left(U^{W}\right)}\right)^{W}$ corresponds to an intensional operator of the category $s / / s$.

The essential point here is a new way of combination of intensional functors with their arguments (another logical structure of intensional contexts). Syntactically two ways of the combination can be presented: $P(a)$ if $P$ is an extensional sign and $\mathbf{Q}[a]$ if $\mathbf{Q}$ is an intensional functor. In these cases methods of calculation of extensions and intensions on semantical level are essentially different. For extensional contexts we have:

(I) the way to determine an intension is $\left(A^{B}\right)^{W} \otimes B^{W} \Rightarrow A^{W}$; so for example $\left(2^{U}\right)^{W} \otimes U^{K} \Rightarrow 2^{W}$ is intension of $P(a)$; (I') $\left(A^{B}\right) \otimes B \Rightarrow$ $A$ - the way to determine extension.

The scheme for intensional contexts is different: 
(II) $\left(A^{B^{W}}\right)^{W} \otimes B^{W} \Rightarrow A^{W}$; so $\left(2^{U^{W}}\right)^{W} \otimes U^{W} \Rightarrow 2^{W}$ is intension of $\mathbf{Q}[a]$; (II') $\left(A^{B^{W}}\right)^{W} \otimes B^{W} \Rightarrow A^{W}$ is the way to determine an extension. So the extension of a complex expression $A[B]$ depends on the intension of the argument expression $B$.

In accordance with two operations of application of functors to their argument two operations of abstraction are used: $\lambda x A$ determines a class of individuals which satisfy the condition $A$, and $\delta x A$ determines a class of individual concepts. Accordingly, different universal quantifiers are introduced [3, pp. 241-243].

By means of simultaneous induction we introduce the concepts of intension of formula and intension of individual expression with respect to function $\varphi$ for evaluation of free individual variables $\operatorname{Int}(A, \varphi)$ and the concept of extension in the world $-\operatorname{Ext}_{H}(A, \varphi)$.

1. $\operatorname{Int}(x, \varphi)=\varphi(x)$;

2. $\operatorname{Ext}_{H}(x, \varphi)=\varphi(x)(H)$;

3. $\operatorname{Ext}_{H}(R, \varphi)=I_{H}(R)$;

4. $\operatorname{Ext}_{H}\left(R\left(\ldots,\lfloor x\rfloor, \ldots x_{j}, \ldots\right), \varphi\right)=t \Leftrightarrow$ $\left\langle\ldots, \varphi(x), \ldots \varphi\left(x_{j}\right)(H) \ldots\right\rangle \in \operatorname{Ext}_{H}(R, \varphi)$;

5. If $A$ is a formula, then $\operatorname{Int}(A, \varphi)=\left\{H \mid \operatorname{Ext}_{H}(A, \varphi)=t ;\right\}$ in other form $\operatorname{Ext}_{H}(A, \varphi)=t \Leftrightarrow H \in \operatorname{Int}(A, \varphi)$;

6. $\operatorname{Int}(A \& B, \varphi)=\operatorname{Int}(A, \varphi) \operatorname{Int}(B, \varphi)$;

7. $\operatorname{Int}(\neg A, \varphi)=W-\operatorname{Int}(A, \varphi)$;

8. $\operatorname{Int}\left(\square_{i} A, \varphi\right)=\left\{H \mid \operatorname{Int}_{H}(A, \varphi) \in \Theta_{i}(H)\right\}$;

9. $\operatorname{Int}(x=y, \varphi)=\{H \mid \varphi(x)(H)=\varphi(y)(H)\}$;

10. $\operatorname{Ext}_{H}(\lambda x A, \varphi)=\left\{m \in U \mid \forall \varphi^{\prime}\left(m=\varphi^{\prime}(x)(H) \Lambda \varphi^{\prime}={ }_{H x} \varphi \Rightarrow\right.\right.$ $\left.\left.H \in \operatorname{Int}\left(A, \varphi^{\prime}\right)\right)\right\}$;

11. $\operatorname{Ext}_{H}(\delta x A, \varphi)=\left\{w \in U^{W} \mid \forall \varphi^{\prime}\left(w=\varphi^{\prime}(x)(H) \Lambda \varphi^{\prime}={ }_{x} \varphi \Rightarrow\right.\right.$ $\left.\left.H \in \operatorname{Int}\left(A, \varphi^{\prime}\right)\right)\right\}$;

12. $\operatorname{Ext}_{H}(\Lambda(\lambda x A), \varphi)=t \Leftrightarrow U \in \operatorname{Ext}_{H}(\lambda x A, \varphi) \Leftrightarrow$ $\forall \varphi^{\prime}\left(\varphi^{\prime}{ }_{H x} \varphi \Rightarrow H \in \operatorname{Int}\left(A, \varphi^{\prime}\right)\right)$;

13. $\operatorname{Ext}_{H}(\Lambda .(\lambda x A), \varphi)=t \Leftrightarrow U_{H} \subseteq \operatorname{Ext}_{H}(\lambda x A, \varphi)$;

14. $\operatorname{Ext}_{H}(\Lambda(\delta x A), \varphi)=t \Leftrightarrow U^{W} \subseteq \operatorname{Ext}_{H}(\delta x A, \varphi)$; 
15. $\operatorname{Ext}_{H}((\lambda x A)(y), \varphi)=t \Leftrightarrow \operatorname{Ext}_{H}(y, \varphi) \in \operatorname{Ext}_{H}(\lambda x A, \varphi)$;

16. $\operatorname{Ext}_{H}((\delta x A)[y], \varphi)=t \Leftrightarrow \operatorname{Int}_{H}(y, \varphi) \in \operatorname{Ext}_{H}(\delta x A, \varphi)$;

The introduced notions of extension and intension correspond with two methods of applying functors to argument.

The quantifiers are introduced as:

$$
\begin{aligned}
\Lambda x A & \rightleftharpoons \Lambda(\lambda x A), \\
\forall x A & \rightleftharpoons \forall(\delta x A), \\
\Lambda . x A & \rightleftharpoons \Lambda .(\lambda x A) .
\end{aligned}
$$

According to Montague, an intensional logic can be at least second order one. Acceptance of two methods of application of functors to their arguments and two operations of abstraction respectively allows to introduce intensional predicates without any intensional operators and to construct the semantics for first-order intensional systems.

The principle of substitution of equals in the form $a=b \supset A(a) \equiv$ $A(b)$ holds in IPL but the principle $a=b \supset A(a) \equiv A(b)$ does not hold.

Proposed approach discovers peculiarities of semantics of intensional contexts and explains why the principle of mutual replacement is violated. It gives the key for comprehension Kripke's puzzle of belief contexts $[1,2]$.

The peculiarities of IPL: (1) any expression including intensional predicates and operators has an intension as well as an extension; (2) intensional contexts differ from extensional by ascription of specific values to intensional predicates (operators) and, what is more important, by a way of their combination with arguments; (3) an intension of any complex extensional expression is a function of intensions of its compounds; (4) an extension of any complex intensional expression is a function of functor's extension and intensions of its arguments; (5) unlike Montague's method, this approach allows to construct an intensional logic as first-order system. 


\section{References}

[1] Kripke, S., A Puzzle about Belief, in A. Margalit (ed.), Meaning and Use, Reidel, 1979.

[2] KRIPKE, S., Outline of a theory of truth, The journal of Philosophy, 92:690-715, 1975.

[3] Smirnova, E. D., On a puzzle about belief, Logical Investigations 8: , 2001 (in Russian).

[4] Smirnova, E. D., Logic in philosophy and philosophical logic. The Edwin Mellen Press New York USA, Queenston, Ontario Canada, 2000 (in Russian).

[5] Smirnova, E. D., Generalizing approach to the construction of logical semantics and its methodological basis, Logical investigations 12:249263, 2005 (in Russian). 